\title{
ANÁLISE DO COMPORTAMENTO APLICADA ÀS DOENÇAS CRÔNICAS NÃO TRANSMISSÍVEIS
}

\author{
APPLIED BEHAVIOR ANALYSIS FOR CHRONIC NONCOMMUNICABLE DISEASES
}

\author{
TAís da COSTA CALHEIROS - ORCID 0000-0001-6319-9852 \\ CONSUltório PARTICULAR DE PSICOlogia CLínICA, BRAsIL \\ MÁRCIA CRISTINA CASERTA GON - ORCID 0000-0002-2389-9587 \\ UNIVERSIDADE ESTADUAL DE LONDRINA, BRASIL
}

\begin{abstract}
RESUMO
No Brasil, os fatores de risco à saúde da população estão fortemente relacionados às Doenças Crônicas Não Transmissíveis [DCNT], as quais apresentam variáveis comportamentais envolvidas na sua etiologia e evolução. O crescente emprego da tecnologia da Análise do Comportamento Aplicada [ACA] tem evidenciado o impacto e a adequação da mesma na resolução de problemas de saúde. Portanto, o objetivo do presente estudo foi conhecer e analisar a produção científica brasileira stricto sensu da ACA, elaborada na última década, concernente a quatro grupos principais de DCNT. As seis dimensões da ACA (Baer, Wolf, \& Risley, 1987) foram identificadas e descritas nessas produções. A estratégia de busca "análise do comportamento" AND saúde foi aplicada em dois bancos de dados nacionais. Estudos empírico-aplicados, escritos por um(a) psicólogo(a) e com delineamento de sujeito único ou de grupo foram analisados descritivo-qualitativamente. Duas dissertações e uma tese foram recuperadas. As dimensões "aplicada" e "analítica e conceitualmente sistemática" foram atendidas, enquanto as demais o foram parcialmente ou não. Verificou-se a urgência do desenvolvimento de pesquisas similares às identificadas neste estudo. A formação de analistas do comportamento, bem como a qualidade da tecnologia que for desenvolvida podem ser beneficiadas pela especificação desse tipo de critério técnico da área.
\end{abstract}

Palavras-chave: análise do comportamento aplicada, saúde, formação científica, tecnologia comportamental.

\begin{abstract}
In Brazil, the risk factors to the health of the population are strongly related to the growth of Chronic Noncommunicable Diseases [CNCDs], which present behavioral variables involved in its etiology and evolution. The increasing use of the Applied Behavior Analysis [ABA] technology has evidenced the impact and adequacy of the same in the resolution of health problems. Therefore, the aim of this study was to know and analyze the Brazilian scientific production in the stricto sensu of the ABA, developed in the last decade, concerning of four main groups of CNCDs. The six dimensions of the ABA (Baer, Wolf, \& Risley, 1987) were identified and described in these productions. The search strategy "behavior analysis" AND health was applied in two national databases. Empirical-applied studies, written by a psychologist and with a design of one subject or group were analyzed descriptive qualitatively. Two dissertations and one thesis were recovered. The dimensions "applied" and "analytical and conceptually systematic" were met, while the others were partially or not. It was verified the urgency of the development of research similar to the ones identified in this study. The training of the behavior analysts as well as the quality of technology that is developed can be benefited by the specification of this type of technical criterion of the area.
\end{abstract}

Keywords: applied behavior analysis, health, scientific training, behavioral technology.

O trabalho foi realizado com apoio financeiro da Coordenação de Aperfeiçoamento de Pessoal de Ensino Superior (Processo 1522850) e da Fundação Araucária (Convênio 062/2013 - Protocolo 33.718).

Correspondência: Taís da Costa Calheiros. E-mail: calheirostais@gmail.com

DOI: http://dx.doi.org/10.18542/rebac.v15i1.8670 
Sabe-se que as principais causas de comprometimento da qualidade de vida e de morte no mundo são as doenças crônicas. No Brasil, os fatores de risco à saúde da população estão fortemente relacionados ao crescimento de Doenças Crônicas Não Transmissíveis [DCNT], as quais correspondem a $72 \%$ das causas de morte (Instituto Brasileiro de Geografia e Estatística [IBGE], 2013), sendo esse o problema de saúde de maior magnitude no país no contexto atual (Ministério da Saúde [MS], 2011). Essas doenças caracterizam-se pela multiplicidade de fatores de risco complexos, interação de fatores etiológicos desconhecidos, danos físicos, manifestações clínicas com períodos de exacerbação, remissão e evolução para incapacidades e morte, além do impacto social e psicológico (Reiners, Azevedo, Vieira, \& Arruda, 2008). São consideradas condições decorrentes do "estilo de vida ocidental" (Achutti \& Azambuja, 2004), cujos fatores de risco são modificáveis, i.e., álcool, tabagismo, alimentação não saudável, obesidade e inatividade física (MS, 2011).

Segundo o Plano de Ações Estratégicas para o Enfrentamento das DCNT no Brasil 2011-2022 (MS, 2011), os quatro grupos principais de DCNT são as doenças circulatórias (i.e., acidente vascular cerebral, infarto, hipertensão arterial), câncer, diabetes e doenças respiratórias crônicas, as quais atingem indivíduos de todas as camadas socioeconômicas e, de forma mais intensa, aqueles com baixa escolaridade e renda, bem como os idosos. Hipertensão e diabetes, e.g., são as condições-chave na determinação da morbimortalidade por DCNT nos próximos anos no Brasil (Duncan et al., 2012). Sendo assim, esses grupos indicados pelo MS (2011) foram identificados como doenças que, atualmente, apresentam extrema necessidade de atenção dos profissionais da área da saúde, inclusive de psicólogos.

$\mathrm{O}$ uso de procedimentos comportamentais, que evidenciem uma fundamentação empírica, na prevenção, na avaliação e no tratamento de doenças tem proliferado (Arruda \& Zannon, 2002; Redd \& Rusch, 1985) e o impacto potencial e a utilidade da tecnologia comportamental na prestação de serviços em saúde são tópicos crescentemente enfatizados (Kerbauy, 2002; Reid, 1991; Smith, Kendall, \& Keefe, 2002). Ademais, têm-se demonstrado a importância da análise científica do comportamento no contexto da saúde, tanto para fins de promoção de comportamentos preventivos, quanto à modificação de comportamentos de risco (Gon, Zakir, Andrade, Gavazzoni, \& Brandenburg, 2015; Moraes \& Rolim, 2012).

No aspecto econômico, as intervenções fundamentadas na Análise do Comportamento Aplicada [ACA] geram a condição de saúde por meio de tratamentos com uma relação custo-benefício vantajosa e efetiva (Kaplan, 1990). Essa ponderação faz-se imprescindível, pois os profissionais da área da saúde têm conhecimento de que as atividades recebem suporte financeiro se forem fornecidas evidências sobre a relação custo-eficácia das propostas de atendimento (Maynard, 1991). Sabe-se, e.g., que $86 \%$ do gasto no setor da saúde, nos Estados Unidos da América [EUA], é direcionado ao manejo de doenças crônicas (Kvedar, Fogel, Elenko, \& Zohar, 2016).

Dado que as DCNT contam com fatores comportamentais na sua etiologia e evolução (Dumith, Muniz, Tassitano, Hallal, \& Menezes, 2012; Guimarães, 1999; Silva, Peres, Boing, González-Chica, \& Peres, 2013; Silva et al., 2014), reconhece-se a possibilidade de intervenção sobre os mesmos (Malta et al., 2015). Em decorrência disso, a ACA têm produzido conhecimento e tecnologia a nível internacional (e.g., Andrade, Barry, Litt, \& Petry, 2014; Dallery, Raiff, \& Grabinski, 2013; Fogel, Miltenberger, Graves, \& Koehler, 2010; Hausman, Borrero, Fisher, \& Kahng, 2014; Kurti \& Dallery, 2013; Larson, Normand, Morley, \& Miller, 2013; Malerbi \& Matos, 2001; Washington, Banna, \& Gibson, 2014).

Em função de o Brasil caracterizar-se como o maior centro de AC depois dos EUA (Todorov \& Hanna, 2010) e da pertinência das pesquisas da ACA diante de problemáticas da área da saúde, hipotetizou-se que uma revisão crítica de estudos brasileiros empírico-aplicados da ACA (i.e., estudos acerca de comportamentos socialmente relevantes baseados na produção de dados empíricos) sobre as DCNT pode oferecer um panorama acerca da consistência técnica dessa produção no país e evidenciar suas contribuições a essa temática. Além disso, ao reconhecer que a formação do psicólogo perpassa pelo desenvolvimento de competências de pesquisa (Resolução n. 8, 2004) e que a universidade apresenta um vínculo formal com a produção de conhecimento científico (Kerbauy, 1999), entendeu-se como essencial analisar dissertações e teses, uma vez que essa atividade pode favorecer para que os analistas do comportamento avaliem o próprio trabalho desenvolvido na área.

Diante desse contexto, o presente trabalho objetivou conhecer e analisar a produção científica brasileira stricto sensu da ACA na área da saúde, produzida no período de 2005 a 2015, com ênfase nos quatro grupos principais de DCNT. Mais especificamente, pretendeu-se descrever as dimensões da ACA (Baer et al., 1987) nessas produções devido ao reconhecimento das mesmas enquanto critérios de avaliação e caracterização desse tipo de intervenção (Moreira, 2005).

\section{MÉTODO}

Trata-se de uma revisão crítica da literatura composta pelas etapas descritas a seguir:

Delimitação da questão a ser pesquisada. Realizou-se um levantamento bibliográfico preliminar no período de 29/02 a 03/03/2016, a fim de investigar a existência de trabalhos de revisão crítica ou de análise da produção científica analítico-comportamental voltados à área da saúde, tendo como critério de avaliação as diretrizes de Baer et al. (1987).

Foi feita uma revisão de literatura em duas bases de dados on-line de referência na área da Psicologia, PsycINFO e Web of Science, e no Portal de Periódicos da Coordenação de Aperfeiçoamento de Pessoal de Nível Superior [CAPES]. 
Os termos utilizados nas estratégias de busca foram identificados a partir do estudo de dois vocabulários controlados: Descritores em Ciências da Saúde [DeCS] da Biblioteca Virtual em Saúde [BVS] e Thesaurus of Psychological Index Terms da PsycINFO. Em função desse recurso não estar disponível em todas as bases de dados eletrônicas, foi feita a combinação de palavraschave e descritores identificados em resumos de trabalhos pertencentes à disciplina da ACA na área da saúde. Essa lista de palavras-chave foi inserida nos vocabulários controlados para verificar quais existiam como descritores exatos.

Notou-se o uso de três terminologias distintas para nomear a ACA no contexto brasileiro, as quais foram empregadas como palavras-chave (keywords) ou tópico (topic), juntamente com o operador booleano AND, os descritores exatos e as demais palavras-chave anteriormente selecionadas, sendo estas: saúde, análise do comportamento, análise aplicada do comportamento, análise do comportamento aplicada, análise comportamental aplicada, revisão, dimensões, behavior analysis, applied behavior analysis, review, dimensions, health care psychology, behavioral medicine, medicine.

As fontes de dados foram consultadas por meio da busca avançada, com exceção da base de dados Web of Science, na qual se optou pela busca simples devido à interface da base. No Portal de Periódicos da CAPES, a busca avançada por assunto foi realizada para que todos os periódicos e repositórios fossem contemplados nesse levantamento bibliográfico preliminar. $\mathrm{O}$ refinamento dos resultados foi realizado posteriormente de forma manual.

Dentre os 639 resumos encontrados, nenhum deles consistia em uma revisão crítica da produção científica brasileira stricto sensu da ACA na área da saúde, principalmente concernente às DCNT, sugerindo a originalidade da proposta. Nesse caso, deu-se início ao desenvolvimento da revisão crítica da literatura propriamente dita.

Escolha das fontes de dados. Foi feito outro levantamento com o intuito de identificar as fontes de dados mais adequadas para a realização da revisão. A partir da seleção de busca avançada por base de dados no Portal de Periódicos da CAPES, com os critérios de Tipo [Teses e Dissertações], Áreas do Conhecimento [Ciências Humanas] e "buscar somente" bases nacionais, foram encontradas 57 bases de dados on-line. Dentre essas, foram selecionadas duas bases de dados de abrangência nacional que disponibilizam o conteúdo de várias Instituições de Ensino Superior [IES] devido ao objetivo dessa pesquisa. A Biblioteca Digital Brasileira de Teses e Dissertações [BDTD] é do Instituto Brasileiro de Informação em Ciência e Tecnologia [IBICT] e o Banco de Teses da CAPES foi criado pelo Ministério da Educação [MEC] para aprimorar os cursos de pósgraduação no contexto brasileiro.

Escolha das palavras-chave para a busca. Foi feito um levantamento por meio da estratégia de busca "análise do comportamento" AND saúde inserida em "busca avançada" no Banco de Teses da CAPES, a partir da seleção de "contém todos os campos" e com o acréscimo de psicologia “é (exato)” como “área de conhecimento". Na BDTD, essa estratégia de busca foi colocada também em "busca avançada", na marcação de "todos os campos", a partir de "todos os termos" e considerando o "ano de publicação" no período de 2005 a 2015.

Realização do levantamento bibliográfico e armazenamento dos resultados. A coleta de dados foi realizada nos dias 08 e 09/03/2016.

Seleção dos estudos pelo resumo, a partir dos critérios de inclusão e de exclusão. Os critérios de inclusão consistiram na dissertação ou tese caracterizar-se como estudo empírico-aplicado da ACA na área da saúde com ênfase em um dos quatro grupos principais de DCNT, com delineamento metodológico de sujeito único ou de grupo, escrita por um(a) psicólogo(a) formado(a) em um dos programas de Mestrado acadêmico ou Doutorado na área de Psicologia no Brasil e cuja data de defesa estivesse no período de 2005 a 2015. Para obter a certificação dessa formação, consultou-se o Currículo Lattes dos pesquisadores.

Foram excluídos resumos: repetidos, incompletos, de outras áreas da saúde (e.g., Medicina, Enfermagem, etc.), de trabalhos teórico-conceituais, de revisões de literatura, de estudos de caso sem delineamento metodológico, de pesquisas relacionadas a transtornos psiquiátricos conforme o Manual Diagnóstico e Estatístico de Transtornos Mentais [DSM-V], da American Psychiatric Association [APA] (2013), bem como aqueles que não eram de autoria de psicólogos, que não utilizavam termos da $\mathrm{AC}$, que não remetiam a um dos quatro grupos principais de DCNT, que não consistiam em produção científica brasileira stricto sensu da área de Psicologia e cuja data de defesa não estava situada entre 2005 e 2015.

Dentre os 68 resumos encontrados, três estudos analítico-comportamentais foram recuperados.

Extração, avaliação e síntese dos dados. A extração dos dados foi feita por meio da leitura na íntegra das produções recuperadas e do registro dos dados em duas planilhas no software Microsoft Excel (versão 2010) elaboradas a partir do Roteiro de Análise da Produção Científica. Esse roteiro foi desenvolvido em formato de perguntas norteadoras do procedimento de análise. As variáveis de estudo que caracterizaram cada dimensão da ACA foram identificadas a partir da descrição conceitual de cada uma delas (Baer et al., 1968, 1987) e das questões propostas por Malavazzi, Malerbi, Del Prette, Banaco e Kovak (2011). Ressalta-se que as contribuições de Baer et al. (1968) se restringiram a aspectos comuns entre as duas versões, porém alguns conceitos estão mais bem detalhados nessa primeira versão.

A escolha da categorização de Baer et al. (1987) ocorreu devido ao argumento de que uma pesquisa deve ser considerada da ACA se demonstrar como realizar as mudanças comportamentais especificadas (analítica) e se o método para promover essa mudança estiver, sistematicamente, relacionado aos princípios básicos da AC (conceitualmente sistemática). Baer et al. (1987) apresentam as dimensões analítica e conceitualmente 
sistemática como complementares e, consequentemente, postulam a junção delas. Salienta-se, com isso, a importância da criação de tecnologias e não apenas de técnicas (Baer et al., 1968). Sumariamente, portanto, para que uma investigação se caracterize como uma análise comportamental aplicada, essa deve contemplar seis dimensões: aplicada, comportamental, analítica e conceitualmente sistemática, tecnológica, efetiva e passível de generalidade.

Elaboração das planilhas no Microsoft Excel. As 20 questões que compõem o instrumento foram inseridas em duas planilhas no Microsoft Excel. Ao lado da coluna com essas perguntas, foram feitas outras três colunas, sendo uma para cada produção stricto sensu. Na planilha das respostas discretas, cada intersecção entre linha e coluna foi composta por uma lista suspensa criada no próprio programa. Esse recurso permite selecionar um determinado item em meio a uma listagem. No caso dessa pesquisa, as opções de resposta foram: sim, não e parcialmente. $\mathrm{Na}$ planilha das questões abertas, cada intersecção foi preenchida descritivamente por meio da inserção das informações coletadas na produção.

Interpretação dos dados conforme um método pré-determinado. A análise descritivo-qualitativa consistiu no preenchimento das duas planilhas para cada produção científica, com o posterior detalhamento dos resultados obtidos em cada dimensão da ACA.

\section{RESULTADOS}

Quanto à análise da Dimensão Aplicada, as pesquisas recuperadas especificaram o comportamentoalvo da intervenção e a relevância social do mesmo, além de mencionarem o local de condução do estudo. Em Amaral (2010), o comportamento-alvo referiu-se a comportamentos de adesão (i.e., falar, responder verbalmente, solicitar informação, buscar suporte emocional, olhar, olhar para outro estímulo e auxiliar na execução do procedimento médico) e concorrentes (i.e., choramingar, chorar, comportar-se de modo nervoso, agredir fisicamente, gritar, movimentar-se até a imobilização, fugir e protestar) emitidos por crianças submetidas à quimioterapia por intermédio de injeção intramuscular. Essa pesquisa foi conduzida no Ambulatório do Setor de Quimioterapia do Hospital Universitário da Região Norte do Paraná [HURNP]. Casseb (2011) visou intervir no seguimento das regras sobre alimentação e atividade física em adultos com diabetes Tipo 2, tendo como local o próprio domicílio dos participantes, e Martins-Silva (2007) planejou intervir no comportamento pós-cirúrgico e na evolução clínica de pacientes submetidos à cirurgia cardíaca aberta em um hospital geral de porte médio do noroeste do Paraná (e.g., enfermarias, apartamentos, etc.).

Os dados obtidos em relação à Dimensão Comportamental mostraram que a seleção do comportamento-alvo foi justificada em todos os trabalhos por meio da fundamentação teórica sobre a relevância do tema e pela apresentação de dados epidemiológicos. Casseb (2011) acrescentou, também, dados sobre o ônus decorrente de doenças crônicas no mundo e Martins-
Silva (2007) comentou a respeito da condição atual do sistema de saúde brasileiro.

Os dados empíricos foram coletados por meio de observação direta e métodos indiretos somente na pesquisa de Martins-Silva (2007); Amaral (2010) utilizou apenas observação direta e Casseb (2011), métodos indiretos. Sobre o tipo de mensuração empregado, Amaral (2010) adotou o registro de frequência de resposta, Casseb (2011) usou o registro de frequência de relato de comportamento e aferição da glicemia e Martins-Silva (2007) utilizou diferentes medidas, i.e., atribuição de nota na avaliação do comportamento-alvo, pontuação atingida em questionário, escore relativo à aplicação de inventário e mensuração do nível de cortisol sanguíneo realizada somente antes da cirurgia cardíaca aberta.

Todos os estudos monitoraram, parcialmente, a atuação das demais pessoas envolvidas na pesquisa, i.e., dos profissionais de saúde, das pesquisadoras e dos familiares ou cuidadores. Ademais, apenas Amaral (2010) utilizou o critério de concordância entre observadores como uma forma de avaliação da fidedignidade dos dados coletados. Em Casseb (2011), foi feita a aferição da glicemia dos participantes e, no caso de Martins-Silva (2007), a aplicação do Inventário de Ansiedade de Beck [BAI], com medida de pré-teste e pós-teste, serviu como critério de comparação entre grupo controle e grupo experimental.

Sobre a Dimensão Analítica e Conceitualmente Sistemática, constatou-se que Amaral (2010) e Casseb (2011) empregaram o delineamento metodológico de sujeito único, tendo sido linha de base múltipla e intrassujeitos e entre condições, respectivamente, enquanto Martins-Silva (2007) utilizou o delineamento experimental entre grupos. Em todas as pesquisas foi demonstrada a relação funcional entre o(s) evento(s) manipulado(s) e o comportamento de interesse, bem como houve o levantamento de hipóteses sobre variáveis intervenientes em detrimento da identificação dessas no próprio procedimento. Sobre o(s) princípio(s) básico(s) da $\mathrm{AC}$, somente Martins-Silva (2007) os especificou.

A Dimensão Tecnológica, de acordo com a qual os procedimentos metodológicos devem ser identificados e descritos de maneira replicável, foi encontrada nos três estudos, mas apenas em Amaral (2010) esse critério foi atendido em sua totalidade. A extensão da variação do procedimento e das condições ambientais que ainda o mantenham efetivo, por sua vez, não foi mencionada nas pesquisas, sendo esse um dos critérios menos atendidos.

A Dimensão Efetiva foi constatada na pesquisa de Martins-Silva (2007), contudo a mudança no comportamento-alvo foi observada parcialmente nas demais produções. Foram realizadas análises da relação custo-benefício da intervenção a partir de parâmetros como: mudanças comportamentais observadas nos participantes, custo financeiro da intervenção, benefícios associados à divulgação do material impresso utilizado na aplicação, recomendações provenientes da literatura científica da área, local onde a pesquisa foi realizada, fidedignidade dos dados coletados, limitações do procedimento e dificuldades enfrentadas pelas 
pesquisadoras durante a condução do trabalho. Em contrapartida, em nenhum dos estudos investigados houve registro quanto à validação social do mesmo.

Sobre os resultados da Dimensão Passível de Generalidade, constatou-se que Casseb (2011) realizou registro de follow-up (i.e., entrevista realizada após um mês), enquanto Amaral (2010) e Martins-Silva (2007) não o fizeram. O processo de generalização de estímulos não foi programado pelas pesquisadoras, mas esse fenômeno foi observado em Casseb (2011), no qual se identificou a ocorrência da generalização de estímulos ao longo do tempo (i.e., após um mês) e entre comportamentos, e.g., usar a medicação e cuidar dos pés.

A partir dos resultados apresentados, pode-se notar que as dimensões "aplicada" e "analítica e conceitualmente sistemática" foram contempladas nas produções analisadas, enquanto as demais foram atendidas parcialmente ou não o foram. Notou-se déficit em relação a: monitoramento do comportamento das demais pessoas envolvidas na pesquisa; identificação e descrição de todos os procedimentos; menção aos princípios básicos que subsidiaram a elaboração do procedimento e à extensão da variação desse e das condições ambientais que mantenham sua efetividade; obtenção de mudança no comportamentoalvo; realização de registros de follow-up e de validação social e programação da generalização.

\section{DISCUSSÃO}

$\mathrm{Na}$ presente revisão crítica, verificou-se que, a partir das estratégias de busca utilizadas, a produção empírico-aplicada de analistas do comportamento, proveniente de cursos de pós-graduação stricto sensu no Brasil, acerca dos quatro grupos principais de DCNT fazse escassa. Essa carência denota a urgência da elaboração de pesquisas da ACA que investiguem os fatores comportamentais envolvidos na ocorrência desse tipo de doença no contexto brasileiro. Ademais, o desenvolvimento desses trabalhos encontra-se concentrado em duas instituições públicas de ensino: Universidade Estadual de Londrina (UEL) e Universidade Federal do Pará (UFPA), nos programas de Mestrado em Análise do Comportamento e de Pós-Graduação em Teoria e Pesquisa do Comportamento, respectivamente. Embora não seja possível fazer uma análise estatística, pode-se hipotetizar que sejam centros formadores de analistas do comportamento na área da saúde no Brasil.

Em relação às seis diretrizes da ACA (Baer et al., 1987), as produções stricto sensu analisadas atenderamnas parcialmente de forma equilibrada, uma vez que, dentre as 20 questões, 10 foram respondidas positivamente. Os aspectos abordados referiram-se a: especificação do local de condução do estudo, do comportamento-alvo, da relevância social e da justificativa da seleção desse comportamento; dados coletados por meio de metodologias diretas e indiretas; descrição do tipo de mensuração e do delineamento metodológico empregados; demonstração da relação funcional entre o(s) evento(s) manipulado(s) e o comportamento de interesse; levantamento de hipóteses sobre variáveis intervenientes e realização de análises da relação custo-benefício da intervenção da ACA.

Um procedimento comumente usado na prática e na pesquisa comportamental aplicada como parâmetro primordial para a avaliação da fidedignidade do que foi mensurado em termos comportamentais diz respeito ao uso do critério de concordância entre observadores (Johnston \& Pennypacker, 2009), o qual não foi constatado em duas produções analisadas. Na pesquisa de Casseb (2011), a aferição da glicemia feita periodicamente não necessita que o critério de concordância entre observadores seja aplicado, pois se trata de uma medida fisiológica diretamente relacionada à variável dependente do estudo, i.e., seguimento das regras sobre alimentação e atividade física. Por outro lado, a não apresentação desse critério em Martins-Silva (2007) não indica uma falha metodológica propriamente dita, mas à impossibilidade de fazê-lo em uma Unidade de Terapia Intensiva devido ao rígido controle do ambiente a que o paciente fica exposto. Em virtude disso, uma reflexão acerca das dificuldades e especificidades à utilização da concordância entre observadores na pesquisa aplicada à área da saúde faz-se pertinente. Pode-se questionar, ainda, se esses aspectos pertencem apenas a essa área ou também a outros contextos de aplicação da ACA. Para tanto, sugere-se a proposição de estudos futuros que investiguem essas questões de pesquisa.

Amaral (2010) e Martins-Silva (2007) observaram, diretamente, o comportamento-alvo, sendo esse o método de mensuração, por excelência, utilizado na ACA ao proporcionar uma avaliação direta, frequente e confiável (Baer et al., 1987). No entanto, Martins-Silva (2007) empregou métodos cujas medidas são diretas e indiretas. Essa conduta pode revelar uma particularidade desse tipo de pesquisa na área da saúde, já que a complexidade do fenômeno estudado requer o uso de diferentes estratégias para a realização de avaliações comportamentais e também porque nem sempre é possível uma mensuração direta do comportamento-alvo. Essa dificuldade relaciona-se, principalmente, à medição de comportamentos privados, i.e., aqueles que só podem ser acessados diretamente pela pessoa que os apresenta e pelos demais mediante relato verbal (Zazula, Sartor, Dias, \& Gon, 2014). Em Martins-Silva (2007), e.g., a "ansiedade" foi investigada por meio da aplicação do BAI.

Apesar de muitos indivíduos apresentarem DCNT, as pesquisas analisadas foram desenvolvidas com um número restrito de participantes, sendo duas crianças, 12 adultos e 20 adultos, em comparação a estudos epidemiológicos conduzidos na área da saúde, o que pode estar associado às peculiaridades da metodologia científica da $\mathrm{AC}$, a qual tem por base metodológica o uso do delineamento de sujeito único em detrimento de métodos estatísticos (Rodrigues \& Moroz, 2008). Embora MartinsSilva (2007) tenha empregado um delineamento de grupo com estatística, Amaral (2010) e Casseb (2011), e.g., monitoraram o desempenho individual dos participantes, quer tenham sido submetidos à mesma intervenção ou a intervenções distintas como membros de grupos em diferentes condições experimentais. 
A Dimensão Tecnológica foi a menos atendida nas pesquisas recuperadas, o que pode comprometer a disseminação adequada dos conhecimentos aplicados a novos contextos devido a falhas no controle de variáveis e na mudança do procedimento (Baer et al., 1987). A referida deficiência técnica prejudica o objetivo científico de replicação e as metas assistenciais da intervenção da ACA pautada em procedimentos claros (Malavazzi et al., 2011). Esse fenômeno pode se relacionar às hipóteses mencionadas por Hayes, Rincover e Solnick (1980) de que a importância e o uso de determinadas dimensões parecem ser tão claros e aparentes, inerentes a um estudo da ACA, que dispensam avaliação ou porque não se pode medi-las de forma confiável. Nota-se, assim, que imprecisões conceituais e obstáculos metodológicos estão presentes, historicamente, na delimitação das características técnicas da ACA. Talvez, por isso, as três autoras não tenham se detido no atendimento e avaliação dessa dimensão.

A mensuração da validação social permite ao pesquisador predizer ou evitar a rejeição da intervenção, sendo crucial à disseminação e sobrevivência da tecnologia gerada. Além disso, o impacto de um estudo não pode ser conhecido antes da sua aplicabilidade ter sido testada no contexto social (Hayes et al., 1980). Dessa forma, a ausência de registros, nas pesquisas analisadas, em relação a esse item interfere na avaliação da efetividade das intervenções, o que evidencia uma demanda de produção de conhecimento para tornar permanente a aplicação, a manutenção e o fortalecimento da ACA no tratamento das DCNT. Já se têm exemplos de pesquisas da ACA destinadas a problemas de saúde pública, e.g., Lyles et al. (2007), que podem servir de base para o desenvolvimento de estratégias similares de manejo das contingências comportamentais que fazem parte desses quadros clínicos.

Um dado positivo a ser destacado diz respeito ao processo de generalização de estímulos ter sido alcançado entre comportamentos por Casseb (2011). Hayes et al. (1980) admite que a ausência da programação do processo de generalização de estímulos é um problema crítico na área, cuja maior parte das pesquisas busca melhorar a demonstração dos efeitos comportamentais da intervenção sem, contudo, alcançar a generalização dos mesmos. Ademais, privilegiam a ocorrência desse fenômeno entre ambientes e no tempo, ao passo que ignoram a generalização entre comportamentos e entre pessoas. Sem esse planejamento, a avaliação da eficácia das tecnologias produzidas torna-se inviável (Malavazzi et al., 2011).

A definição de pesquisa aplicada apresentada por Baer et al. (1968, 1987) já foi rotulada como "estrutural" em detrimento da definição funcional (Hayes et al., 1980). Segundo Hayes et al. (1980), uma pesquisa aplicada deve corresponder a qualquer estudo que aumente a habilidade dos "aplicadores" em entender, predizer e controlar comportamentos socialmente relevantes nos contextos e com os clientes a que servem. Nesse sentido, as regras que permeiam a condução de pesquisas e intervenções da ACA estariam pouco claras? Esse direcionamento deveria ser periodicamente exposto e discutido por comportamentalistas brasileiros, tal como foi feito por Moreira (2005) e Malavazzi et al. (2011)?

Diante da complexidade da definição do desenho técnico da ACA (Hayes et al., 1980), seria importante e recomendável que pesquisadores, ao proporem estudos dessa natureza, procurassem organizá-los, metodologicamente, a partir desses critérios e, quando isso não fosse possível ou que esses não tenham sido atendidos, que considerações esclarecedoras sobre cada uma das dimensões fossem feitas. Essas informações viabilizariam averiguar se tais dificuldades são inerentes à própria definição das dimensões (Hayes et al., 1980), à maneira como essas estão sendo adotadas pelos pesquisadores da área ou se estão relacionadas à adequação ao contexto de pesquisa e/ou à realidade brasileira.

Sob outra perspectiva, o conhecimento das dimensões da ACA de modo mais sistematizado poderia levar pesquisadores brasileiros a aprimorarem ainda mais suas produções de modo a aumentar a probabilidade de terem os seus trabalhos aceitos em periódicos internacionais de maior impacto, mais especificamente na área das DCNT. Para tanto, sugere-se o refinamento do roteiro de análise proposto nesta revisão mediante a elaboração de pesquisas futuras que avaliem a adequação, a suficiência e a precisão das variáveis e da metodologia de registro utilizada. Indica-se, ainda, a investigação de possíveis circunstâncias que possam interferir para a conformidade das diretrizes da ACA em pesquisas empírico-aplicadas na área da saúde e que incluam outras condições médicas além das DCNT. Esse estudo poderia ser conduzido por meio de instrumentos previamente elaborados com esse objetivo e destinados a analistas do comportamento, psicólogos ou não, que produzem conhecimento científico da ACA na área da saúde em IES no Brasil.

\section{CONSIDERAÇÕES FINAIS}

Compreender os fatores associados aos cuidados com a saúde tem sido um dos objetivos primordiais de estudiosos interessados no avanço do tratamento de doenças crônicas (Ferreira, 2001). No entanto, no Brasil, ainda não são tão frequentes estudos que examinem as características da produção científica teórica e aplicada da Psicologia e da Análise do Comportamento a partir de uma investigação minuciosa, o que evidencia a relevância desse tipo de pesquisa tida como "histórica" (Rodrigues \& Moroz, 2008).

$\mathrm{O}$ presente estudo procurou verificar o que se produziu de ACA em cursos de pós-graduação stricto sensu no Brasil, no período de 2005 a 2015, em relação às doenças circulatórias, respiratórias crônicas, câncer e diabetes. Por meio das estratégias de busca utilizadas, notou-se que, embora limitada quantitativamente e em abrangência, a literatura científica recuperada agrega algumas contribuições da ACA produzidas, recentemente, no contexto brasileiro para o enfrentamento das DCNT. No entanto, devido, principalmente, ao déficit no atendimento dos critérios circunscritos à dimensão tecnológica, vislumbra-se que a eficácia dos procedimentos, bem como a apropriação e a disseminação 
da ACA junto a pacientes, familiares, cuidadores e/ou profissionais de saúde podem estar comprometidas, o que referendaria a afirmação de que "a Análise do Comportamento Aplicada parece cumprir, parcialmente, sua finalidade hoje" (Malavazzi et al., 2011, p. 226).

A especificação de direcionamentos técnicos a serem considerados na análise e na produção de conhecimento empírico da ACA pode aprimorar a formação científica de analistas do comportamento dedicados à interface comportamento e saúde, concedendo parâmetros para que se tenha o devido rigor conceitual e metodológico nessa atividade. Com profissionais preparados para fazer ciência pautada nos fundamentos teórico-metodológicos da ACA, pode-se colaborar à qualidade e pertinência da tecnologia analíticocomportamental desenvolvida para intervir diante de problemas de saúde como os são as DCNT no Brasil.

\section{DECLARAÇÃO DE CONFLITO DE INTERESSES}

Os autores declaram que não há conflito de interesses relativos à publicação do presente artigo.

\section{CONTRIBUIÇÃO DE CADA AUTOR}

Certificamos que todos os autores participaram suficientemente do trabalho para tornar pública sua responsabilidade pelo conteúdo conforme aqui especificado: T. C. C. participou na coleta, tabulação e análise dos dados; redação do manuscrito e de sua versão final. M. C. C. G. participou das etapas do trabalho como orientadora e na redação e revisão crítica do conteúdo e versão final do manuscrito.

\section{DIREITOS AUTORAIS}

Este é um artigo aberto e pode ser reproduzido livremente, distribuído, transmitido ou modificado, por qualquer pessoa desde que usado sem fins comerciais. O trabalho é disponibilizado sob a licença Creative Commons 4.0 BY-NC.

\section{(cc) $\mathrm{BY}-\mathrm{NC}$}

\section{REFERÊNCIAS}

Achutti, A., \& Azambuja, M. I. R. (2004). Doenças crônicas não-transmissíveis no Brasil: Repercussões do modelo de atenção à saúde sobre a seguridade social. Ciência \& Saúde Coletiva, 9, 833-840. doi: 10.1590/S1413-81232004000400002

* Amaral, M. (2010). Efeitos de uma intervenção comportamental com crianças durante injeção intramuscular para quimioterapia (Dissertação de mestrado, Universidade Estadual de Londrina). Recuperado de http://www.uel.br/

American Psychiatric Association [APA] (2013). Diagnostic and statistical manual of mental disorders (5th ed.). Arlington. doi:

10.1176/appi.books.9780890425596

1 As referências que apresentam asterisco correspondem àquelas analisadas nessa pesquisa.
Andrade, L. F., Barry, D., Litt, M. D., \& Petry, N. M. (2014). Maintaining high activity levels in sedentary adults with a reinforcement-thinning schedule. Journal of Applied Behavior Analysis, 47, 523-536. doi: 10.1002/jaba.147

Arruda, P. M., \& Zannon, C. M. L. C. (2002). Tecnologia comportamental em saúde: Adesão ao tratamento pediátrico da doença crônica: Evidenciando o desafio enfrentado pelo cuidador. Santo André: ESETec.

Baer, D. M., Wolf, M. M., \& Risley, T. R. (1968). Some current dimensions of Applied Behavior Analysis. Journal of Applied Behavior Analysis, 1, 91-97. doi: 10.1901/jaba.1968.1-91

Baer, D. M., Wolf, M. M., \& Risley, T. R. (1987). Some still-current dimensions of Applied Behavior Analysis. Journal of Applied Behavior Analysis, 20, 313-327. doi: 10.1901/jaba.1987.20-313

*Casseb, M. da S. (2011). Efeito de três procedimentos de intervenção sobre adesão ao tratamento em adultos com diabetes (Tese de doutorado, Universidade Federal do Pará). Recuperado de http://ppgtpc.propesp.ufpa.br/

Dallery, J., Raiff, B. R., \& Grabinski, M. J. (2013). Internet-based contingency management to promote smoking cessation: A randomized controlled study. Journal of Applied Behavior Analysis, 46, 750-764. doi: $10.1002 /$ jaba.89

Dumith, S. C., Muniz, L. C., Tassitano, R. M., Hallal, P. C., \& Menezes, A. M. (2012). Clustering of risk factors for chronic diseases among adolescents from Southern Brazil. Preventive Medicine, 54, 393-396. doi: 10.1016/j.ypmed.2012.03.014

Duncan, B. B., Chor, D., Aquino, E. M. L., Bensenor, I. M., Mill, J. G., Schmidt, M. I., Lotufo, P. A., Vigo, A., \& Barreto, S. M. (2012). Doenças crônicas não transmissíveis no Brasil: Prioridade para enfrentamento e investigação. Revista de Saúde Pública, 46, 126-134. doi: 10.1590/S0034-89102012000700017

Ferreira, E. A. P. (2001). Adesão ao tratamento em portadores de diabetes: Efeitos de um treino em análise de contingência sobre comportamento de autocuidado (Tese de doutorado, Universidade de Brasília). Recuperado de http://bases.bireme.br/

Fogel, V. A., Miltenberger, R. G., Graves, R., \& Koehler, S. (2010). The effects of exergaming on physical activity among inactive children in a physical education classroom. Journal of Applied Behavior Analysis, 43, 591-600. doi: 10.1901/jaba.2010.43-591

Gon, M. C. C., Zakir, N. S., Andrade, L. V., Gavazzoni, J. A., \& Brandenburg, O. J. (2015). Possíveis relações entre comportamento e condição de saúde. In V. B. Haydu \& S. R. Souza (Orgs.), Análise do Comportamento aplicada a diferentes contextos (pp. 75-94). Londrina: EDUEL.

Guimarães, S. S. (1999). Psicologia da Saúde e doenças crônicas. In R. R. Kerbauy (Org.), Comportamento $e$ saúde: Explorando alternativas (pp. 22-45). Santo André: ARBytes.

Hausman, N. L., Borrero, J. C., Fisher, A., \& Kahng, S. (2014). Improving accuracy of portion-size estimations 
through a stimulus equivalence paradigm. Journal of Applied Behavior Analysis, 47, 485-499. doi: 10.1002/jaba.139

Hayes, S. C., Rincover, A., \& Solnick, J. V. (1980). The technical drift of Applied Behavior Analysis. Journal of Applied Behavior Analysis, 13, 275-285. doi: 10.1901/jaba.1980.13-275

Instituto Brasileiro de Geografia e Estatística [IBGE]. (2013). Pesquisa Nacional de Saúde. Recuperado de http://www.ibge.gov.br/

Johnston, J. M., \& Pennypacker, H. S. (2009). Strategies and tactics of behavioral research (3rd ed.). New York: Routledge.

Kaplan, R. M. (1990). Behavior as the central outcome in health care. American Psychologist, 45, 1211-1220. doi: 10.1037/0003-066X.45.11.1211

Kvedar, J. C., Fogel, A. L., Elenko, E., \& Zohar, D. (2016). Digital medicine's march on chronic disease. Nature Biotechnology, 34, 239-246. doi: 10.1038/nbt.3495

Kerbauy, R. R. (1999). O papel da universidade e a formação do psicólogo que trabalha com comportamento e saúde. In R. R. Kerbauy (Org.), Comportamento e saúde: Explorando alternativas (pp. 10-21). Santo André: ARBytes.

Kerbauy, R. R. (2002). Comportamento e saúde: Doenças e desafios. Psicologia USP, 13, 11-28. doi: 10.1590/S0103-65642002000100002

Kurti, A. N., \& Dallery, J. (2013). Internet-based contingency management increases walking in sedentary adults. Journal of Applied Behavior Analysis, 46, 568-581. doi: 10.1002/jaba.58

Larson, T. A., Normand, M. P., Morley, A. J., \& Miller, B. G. (2013). A functional analysis of moderate-tovigorous physical activity in young children. Journal of Applied Behavior Analysis, 46, 199-207. doi: 10.1002/jaba.8

Lyles, C. M., Kay, L. S., Crepaz, N., Herbst, J. H., Passin, W. F., Kim, A. S., Rama, S. M., Thadiparthi, S., DeLuca, J. B., \& Mullins, M. M. (2007). Best-evidence interventions: Findings from a systematic review of HIV behavioral interventions for US populations at high risk, 2000-2004. American Journal of Public Health, 97, 133-143. doi: 10.2105/AJPH.2005.076182

Malavazzi, D. M., Malerbi, F. E. K., Del Prette, G., Banaco, R. A., \& Kovac, R. (2011). Análise do Comportamento Aplicada: Interface entre ciência e prática? Perspectivas em Análise do Comportamento, 2, 218-230. Recuperado de http://media.wix.com/

Malerbi, F. E. K., \& Matos, M. A. (2001). Blood glucose discrimination training: The role of internal and external cues. Journal of Health Psychology, 6, 229. 240. doi: 10.1177/135910530100600209

Malta, D. C., Iser, B. P. M., Santos, M. A. S., Andrade, S. S. de A., Stopa, S. R., Bernal, R. T. I., \& Claro, R. M. (2015). Estilos de vida nas capitais brasileiras segundo a Pesquisa Nacional de Saúde e o Sistema de Vigilância de Fatores de Risco e de Proteção para Doenças Crônicas Não Transmissíveis por Inquérito Telefônico (Vigitel), 2013. Revista Brasileira de
Epidemiologia, $\quad 18, \quad 68-82 . \quad$ doi: $\quad 10.1590 / 1980-$ 5497201500060007

Maynard, A. (1991). The relevance of health economics to health promotion. In B. Badura \& I. Kickbusch (Eds.), Health promotion research: Towards a new social epidemiology (pp. 29-54). Recuperado de http://www.euro.who.int

* Martins-Silva, M. E. (2007). Efeitos de uma intervenção breve em preparo psicológico pré-cirúrgico na evolução clínica de portadores de cardiopatia (Dissertação de mestrado, Universidade Estadual de Londrina). Recuperado de http://www.uel.br/

Ministério da Saúde [MS]. (2011). Plano de ações estratégicas para o enfrentamento das doenças crônicas não transmissíveis (DCNT) no Brasil. Recuperado de http://bvsms.saude.gov.br/

Moraes, A. B. A., \& Rolim, G. S. (2012). Algumas reflexões analítico-comportamentais na área da Psicologia da Saúde. In N. B. Borges \& F. A. Cassas (Orgs.), Clínica analítico-comportamental: Aspectos teóricos e práticos (pp. 287-293). Porto Alegre: Artmed.

Moreira, E. G. (2005). O Journal of Organizational Behavior Management como um veículo para a publicação de pesquisa em Análise Aplicada do Comportamento, na área de Organizational Behavior Management. (Dissertação de mestrado, Pontifícia Universidade Católica de São Paulo).

Redd, W. H., \& Rusch, F. R. (1985). Behavioral analysis in Behavioral Medicine. Behavior Modification, 9, 131-154. doi: 10.1177/01454455850092001

Reid, D. H. (1991). Technological behavior analysis and societal impact: A human services perspective. Journal of Applied Behavior Analysis, 24, 437-439. doi: 10.1901/jaba.1991.24-437

Reiners, A. A. O., Azevedo, R. C. S., Vieira, M. A., \& Arruda, A. L. G. (2008). Produção bibliográfica sobre adesão/não-adesão de pessoas ao tratamento de saúde. Ciência \& Saúde Coletiva, 13, 2299-2306. doi: 10.1590/S1413-81232008000900034

Resolução n. 8, de 7 de maio de 2004. (2004). Institui as Diretrizes Curriculares Nacionais para os cursos de graduação em Psicologia. Diário Oficial da União, Brasília: CNE/CES.

Rodrigues, M. E., \& Moroz, M. (2008). Formação de professores e Análise do Comportamento: A produção da pós-graduação nas áreas de Psicologia e Educação. Acta Comportamentalia, 16, 347-378. Recuperado de http://www.journals.unam.mx/

Silva, D. A., Peres, K. G., Boing, A. F., González-Chica, D. A., \& Peres, M. A. (2013). Clustering of risk behaviors for chronic noncommunicable diseases: A population-based study in Southern Brazil. Preventive Medicine, $\quad 56, \quad 20-24 . \quad$ doi: 10.1016/j.ypmed.2012.10.022

Silva, K. S., Barbosa Filho, V. C, Del Duca, G. F., Peres, M. A. A., Mota, J., Lopes, A. S., \& Nahas, M. V. (2014). Gender differences in the clustering patterns of risk behaviours associated with non-communicable diseases in Brazilian adolescents. Preventive Medicine, 
65, 77-81. doi: 10.1016/j.ypmed.2014.04.024

Smith, T. W., Kendall, P. C., \& Keefe, F. J. (2002). Behavioral Medicine and Clinical Health Psychology: Introduction to the special issue, a view from the decade of behavior. Journal of Consulting and Clinical Psychology, 70, 459-462. doi: 10.1037/0022006X.70.3.459

Todorov, J. C., \& Hanna, E. S. (2010). Análise do Comportamento no Brasil. Psicologia: Teoria $e$ Pesquisa, 26, 143-153. doi: 10.1590/S010237722010000500013

Washington, W. D., Banna, K. M., \& Gibson, A. L. (2014). Preliminary efficacy of prize-based contingency management to increase activity levels in healthy adults. Journal of Applied Behavior Analysis, 47, 231-245. doi: 10.1002/jaba.119

Zazula, R., Sartor, M. S., Dias, N. G., \& Gon, M. C. C. (2014). Uso de medidas diretas e indiretas para avaliação de problemas de comportamento em crianças com dermatite atópica. In V. B. Haydu, S. A. Fornazari, \& C. R. Estanislau (Orgs.), Psicologia e Análise do Comportamento: Conceituações $e$ aplicações à educação, organizações, saúde e clínica (pp. 267-287). Londrina: UEL.

Submetido em: 29/03/2019

Aceito em: 02/03/2020 